\title{
Indomethacin-Related Leukocytoclastic Vasculitis: A Case Report and Review of Literature
}

\author{
Nasir Hussain Usman Mustafa James Davis Shivani Thakkar \\ Alaa M. Ali Aibek E. Mirrakhimov Aram Barbaryan \\ Guy Anthony Rowley \\ Department of Internal Medicine, Saint Joseph Hospital, Chicago, Ill., USA
}

\section{Key Words}

Indomethacin · Leukocytoclastic vasculitis · Ceretec scan

\begin{abstract}
Leukocytoclastic vasculitis (LCV) is a small-vessel vasculitis with a reported incidence rate of 30 cases per million persons per year. It usually presents as a palpable purpuric skin rash on legs, though any part of the body can be affected. LCV rash may have an associated burning sensation or pain and in some cases may involve internal organs. In some cases, LCV rash may present as nodules, recurrent ulcerations or asymptomatic lesions. The diagnosis of LCV is usually made on skin biopsy. Etiological triggers may not be identified in as many as half of the cases. Treatment is usually conservative and includes identification and removal or treatment of the etiological trigger except in cases with internal organ involvement where systemic steroids and immunosuppressant may be necessary. In this article we present a case of indomethacin-associated LCV that improved with discontinuation of the offending agent.
\end{abstract}

\section{Introduction}

Leukocytoclastic vasculitis (LCV) (hypersensitivity vasculitis) is a term commonly used to denote a small-vessel vasculitis. Many possible causes or associations have been proposed for LCV, but a cause or an associated disorder may not be found in as many as half of the cases.

LCV presents clinically as a cutaneous disease with or without internal body organ involvement. The prognosis of LCV is usually good if internal organs are not affected. The internal organs most commonly involved in LCV are the joints, gastrointestinal tract, and the 
kidneys. Clinical presentation of LCV varies from an acute self-limiting episode to recurrent or chronic forms.

In this article we report a case of a patient who developed painful skin lesions identified to be LCV almost one and a half week after the use of indomethacin. There have been few other reports of LCV secondary to use of nonsteroidal anti-inflammatory drugs. To the best of our knowledge indomethacin-related LCV has been reported only once in the literature.

\section{Case Presentation}

A 50-year-old African-American male with a past medical history of hypertension, diabetes mellitus, peripheral vascular disease, end stage renal disease (ESRD) on hemodialysis (HD) and osteoarthritis presented for evaluation of coccyx bone pain for one week. The pain was sudden in onset, non-radiating and progressively got worse to the point $(10 / 10$ in severity) that patient was wheelchair bound for one week and had missed three consecutive sessions of HD. Patient reported history of a fall few days prior to onset of pain. There was no history of coccyx bone swelling, erythema, drainage, fever or chills. Patient had a history of recurrent methicillin-sensitive Staphylococcus aureus (MSSA) bacteremia; recent MSSA bacteremia episode was almost 2 months prior to the presentation and was due to catheterrelated infection. Rest of review of symptoms was negative. Social history was significant for cigarette smoking (half pack per day for 20 years). At the time of presentation patient was taking aspirin, digoxin, ferrous sulfate, erythropoietin, insulin glargine, insulin lispro, metoprolol, magnesium oxide, pantoprazole, renal multivitamins, tramadol, colchicine (for recurrent pericarditis), senna-docusate and coumadin. Vitals at the time of presentation were: blood pressure of $124 / 70$; pulse 110 per minute, temperature $98.7^{\circ} \mathrm{F}$, respiratory rate of 18 per minute. Patient was 5 feet 10 inches tall and weighed 290 pounds. Physical examination revealed an obese male in mild distress; lungs were clear to auscultation; heart rhythm was regular, no murmur, rub or gallop. Other significant physical examination findings included presence of right upper extremity arteriovenous grafts, healed surgical scar at site of previous arteriovenous graft on left antecubital fossa, permacath right upper chest, right sacroiliac joint tenderness and point tenderness of first sacral vertebrae. Initial diagnostic workup including complete blood count and comprehensive metabolic panel revealed anemia with a hemoglobin of $7.8 \mathrm{~g} / \mathrm{dl}$ (13.5-17.0), hyponatremia with a sodium of $129 \mathrm{mmol} / \mathrm{l}$ (135-145 mmol/l). White blood cell (WBC) and platelet counts were within normal limits; blood urea nitrogen and serum creatinine levels were elevated. Lumbosacral $\mathrm{X}$-ray showed degenerative joint disease without any other significant abnormality. Working diagnosis of sacroiliitis was considered and patient was started on indomethacin $50 \mathrm{mg}$ three times a day for anti-inflammatory and analgesic purposes along with opiate analgesics as needed. Blood culture on day 5 grew Gram-positive cocci later identified as MSSA, infection was thought to be secondary to permacath infection which was removed and the right arm graft was determined to be mature for the HD. Patient was given one dose of vancomycin followed by daily cefazolin. Patient continued to have excruciating sacroiliac and coccygeal bone pain despite of judicious use of analgesics. MRI followed by CT scan of pelvis was done on day 10 and was suggestive of possible sacral osteomyelitis. Interventional radiology team performed CT-guided aspiration of the sacroiliac joint space which returned frank pus, sample was sent for Gram stain and culture. Gram-positive cocci in clusters were identified which were later identified as MSSA. Considering history of recurrent MSSA bacteremia Ceretec-tagged WBC scan was done on day 12 which did not reveal any source of the infection. The following day after Ceretec-tagged WBC scan patient 
was noted to have multiple erythematous painful lesions around wrist area and violaceous erythematous papules involving metacarpophalangeal joints which progressively became generalized (as shown in fig. 1). Skin rash was thought to be a result of septic embolism or an allergic reaction to medicine or Ceretec scan. Skin punch biopsy was performed which demonstrated neutrophils surrounding and infiltrating the wall of the blood vessel, fibrin deposition in the walls of the blood vessels and loss of the endothelial cells which confirmed the diagnosis of the LCV (fig. 2). Indomethacin was discontinued on day 14 and a subsequent improvement and complete resolution of the rash was noted within a week. Additional workup including ANA, RF, ANCA antibodies, complement levels, HIV antibodies and hepatitis B and C serology was within normal limits. Subsequently transthoracic echocardiogram was negative for any evidence of infective endocarditis. Patient had a complicated course of stay but responded well to intravenous antibiotics and was discharged in a stable condition.

\section{Discussion}

LCV is a small-vessel hypersensitivity vasculitis with a reported incidence rate of about 30 cases per million people per year [1]. LCV can occur at any age group and is thought to effect men and women in equal numbers but few studies suggest male predominance [2]. Multiple etiologic factors including drugs, infections, foods, autoimmune diseases, collagen vascular diseases and malignancies have been suggested to associate with LCV [3-6]. Though exact pathogenic mechanism of LCV remains to be elucidated, circulating immune complexes are believed to be involved in the pathogenesis of LCV [7].

LCV usually presents as a palpable purpuric rash associated with burning sensation or pain and is most commonly observed on the legs, but any surface may be involved. Rarely the presentation may include completely asymptomatic lesions, nodular lesions or ulcerations. Thorough history of recent infections, change in medications or food etc. along with a detailed physical examination to find etiologic trigger or an associated disorder should be performed. Identification and avoidance or treatment of the etiologic trigger may prevent recurrent episodes of LCV.

Diagnosis of LCV is confirmed on histological examination of the sample obtained on biopsy of the affected area. Histologically, LCV demonstrates perivascular and vascular leukocytic infiltrates along with fibrinoid necrosis. Etiological triggers are usually identified with the temporal association.

In our patient, indomethacin was started at the day of presentation; one dose of intravenous vancomycin was given on day 5 followed by daily intravenous cefazolin; Ceretec-tagged WBC scan was done one day prior to onset of LCV rash. Considering patient had history of recurrent MSSA bacteremia and had been on vancomycin and cefazolin multiple times, chances that cefazolin or vancomycin was involved in the pathogenesis of LCV were less likely. Furthermore cefazolin was continued throughout the course of the treatment and rash resolved while patient was still on cefazolin. Since indomethacin was the only new medicine to which the patient was recently exposed to, it was thought to be the etiologic trigger for LCV in this case. Further, the LCV rash improved after the discontinuation of the indomethacin confirming our hypothesis. Gamboa et al. [8] described a case of a 22-year-old male who had three episodes of recurrent LCV which coincided with the administration of indomethacin on each occasion. Our patient had onset of LCV rash on the following day after the Ceretec-tagged WBC scan which suggests that technetium-99m might have acted as a trigger for LCV. Acute allergic rash secondary to Tc- $99 \mathrm{~m}$ have been reported to occur in less 
than $1 \%$ of patients but no association for LCV has been described so far in literature and our case may represent the first case of possible Tc-99m-induced LCV.

Treatment of LCV is identification and removal or treatment of the offending etiologic factor, and elevation of the leg and use of compression stocking if LCV rash involves dependent areas. Colchicine [9] and dapsone [10] have been shown to be useful in cases of LCV. Urticarial lesions can be managed with the antihistaminic medicines. Systemic steroids and other immunosuppressive agents [11] may be used in cases of deep organ involvement.

In conclusion, identification of potential etiological triggers in cases of LCV can prevent significant morbidity related with recurrences. Multiple NSAIDs including indomethacin can act as a potential trigger for LCV. Tc-99m may have a role as an etiologic trigger for LCV.

\section{Disclosure Statement}

There is no conflict of interest.

\section{References}

1 Garcia-Porrua C, Gonzalez-Gay MA: Comparative clinical and epidemiological study of hypersensitivity vasculitis versus Henoch-Schönlein purpura in adults. Semin Arthritis Rheum1999;28:404-412.

-2 Gonzalez-Gay MA, Garcia-Porrua C: Systemic vasculitis in adults in northwestern Spain, 1988-1997. Clinical and epidemiologic aspects. Medicine 1999;78:292-308.

3 Sams WM: Hypersensitivity angiitis. J Invest Dermatol 1989;93:78S-81S.

-4 Zurada JM, Ward KM, Grossman ME: Henoch-Schonlein purpura associated with malignancy in adults. J Am Acad Dermatol 2006;55:S65-S70.

5 Solans-Laque R, Bosch-Gil JA, Perez-Bocanegra C, Selva-O Callaghan A, Simeon-Aznar CP, Vilardell-Tarres M: Paraneoplastic vasculitis in patients with solid tumors: report of 15 cases. J Rheumatol 2008;35:294-304.

-6 Fain 0, Hamidou M, Cacoub P, Godeau B, Wechsler B, Paries J, et al: Vasculitides associated with malignancies: analysis of sixty patients. Arthritis Rheum 2007;57:1473-1480.

7 Mackel SE, Jordon RE: Leukocytoclastic vasculitis. A cutaneous expression of immune complex disease. Arch Dermatol 1982;118:296-301.

-8 Gamboa PM, Tabar AI, Wong E: Indomethacin induced vasculitis. Allergol Immunopathol 1988;16:53-55.

-9 Sais G, Vidaller A, Jucgla A, Gallardo F, Peyri J: Colchicine in the treatment of cutaneous leukocytoclastic vasculitis. Results of a prospective, randomized controlled trial. Arch Dermatol 1995;131:1399-1402.

$\checkmark 10$ Wolf R, Tuzun B, Tuzun Y: Dapsone: unapproved uses or indications. Clin Dermatol 2000;18:37-53.

11 Keogh KA, Ytterberg SR, Fervenza FC, Carlson KA, Schroeder DR, Specks U: Rituximab for refractory Wegener's granulomatosis: report of a prospective, open-label pilot trial. Am J Respir Crit Care Med 2006;173:180-187. 


\section{Case Reports in Dermatology}
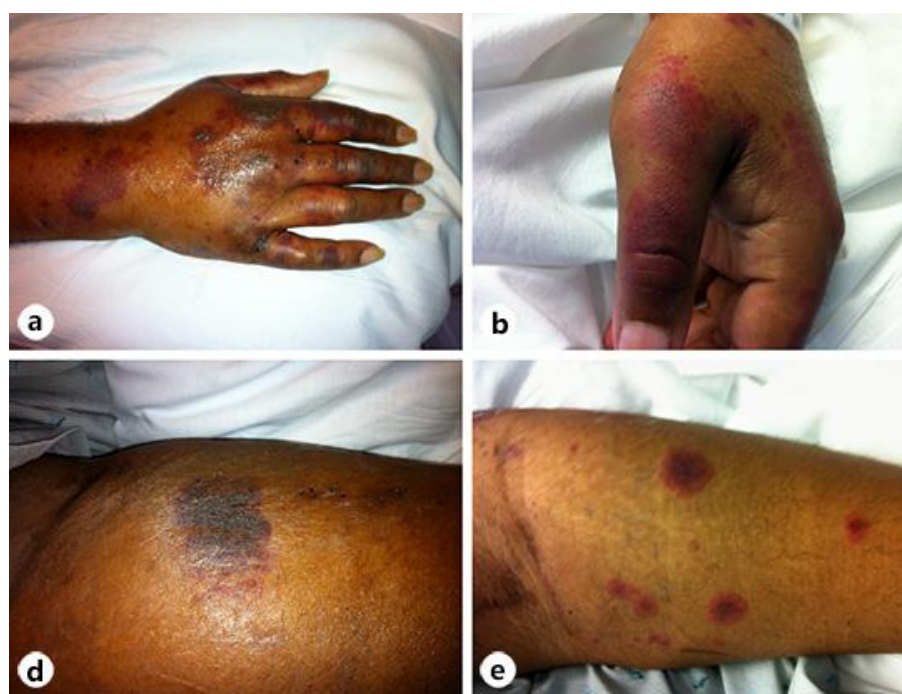

\begin{tabular}{l|l}
\hline Case Rep Dermatol 2013;5:33-37 & \\
\hline DOI: $\underline{10.1159 / 000348240}$ & $\begin{array}{l}@ 2013 \text { S. Karger AG, Basel } \\
\text { www.karger.com/cde }\end{array}$ \\
\hline
\end{tabular}

Hussain et al.: Indomethacin-Related Leukocytoclastic Vasculitis: A Case Report and Review of Literature

Fig. 1. LCV rash on various parts of the body. a, b Rash affecting hands and wrists bilaterally. c-e Rash involving both lower extremities: d shows rash effecting right buttock, c and e show rash effecting bilateral popliteal and calf regions. $\mathbf{f}$ Facial involvement.
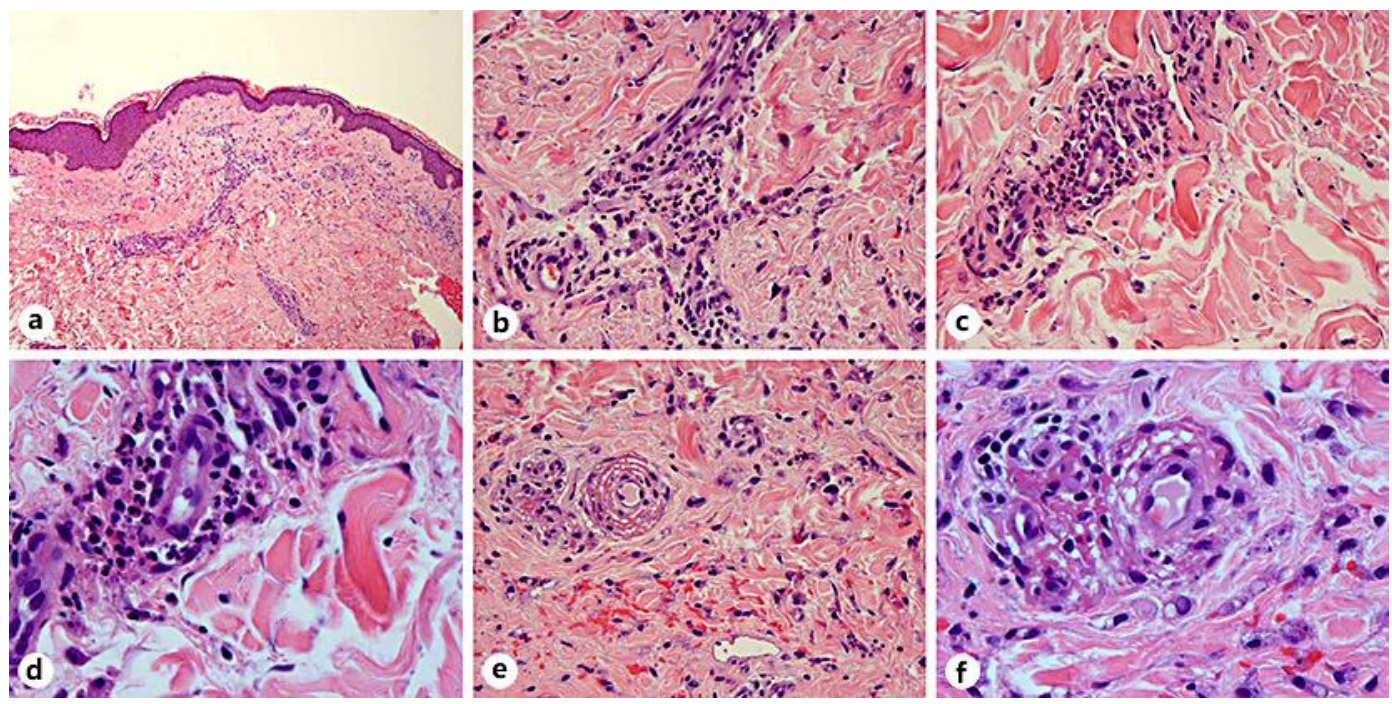

Fig. 2. a Inflammatory cells surrounding blood vessels in dermis $(4 \times)$. b, c (10x), d (20x) Neutrophils surround and infiltrate blood vessel wall, $\mathbf{d}$ also shows loss of endothelial cells. e, $\mathbf{f}$ Small blood vessels with fibrin deposition in wall and neutrophils (10x and 40x, respectively); $\boldsymbol{f}$ also shows loss of endothelial cells consistent with vasculitis. 